\title{
Flow Field Investigation around Body Tail Projectile
}

\author{
Mahfouz Elnaggar Wessam, Zhihua Chen*, Zhengui Huang \\ Key Laboratory of Transient Physics, NUST, Nanjing 210094, Jiangsu, China \\ Email: ${ }^{*}$ chenzh@mail.njust.edu.cn, 1851924502@qq.com
}

Received February 2014

\begin{abstract}
The unsteady compressible flow around a $50 \mathrm{~mm}$ projectile governed by the Navier-Stocks (NS) equation is numerically solved with a Large Eddy Simulation (LES) method, with the Sub-Grid Scale (SGS) solved by Smagorinsky-Lilly model. The computed results are obtained in supersonic flow regime for a viscous fluid in order to determine the aerodynamic coefficients with different angles of attack. The flow around a body tail projectile was solved as a three-dimensional flow.
\end{abstract}

\section{Keywords}

Body Tail Projectile, Aerodynamic Coefficients, Viscosity, Flow Field

\section{Introduction}

The flow around a projectile presents turbulent boundary layers, whose separation is a usual phenomena and a large turbulent wake formed at the bottom of the object. In ballistic aerodynamics, prevention or control of the separation of the boundary layer is one of the most important aims, as well as an appropriate ogive design [1] [2]. As it is well known, a turbulent flow carries irregular and fluctuating fluid motions which contribute significantly to the transport phenomena. They are always three-dimensional, unsteady and mainly irregular except perhaps by coherent structures, which are as some kind of organized flow motion that can be recognized in the instantaneous flow fields as well as in the time-averaged ones [3]. There are also eddies with a wide spectrum of sizes, from the larger ones close to the flow domain ones, to the much smaller ones at which viscous dissipation takes place. The numerical techniques available in Computational Fluid Dynamics (CFD) to simulate them can be split in three main types [4] [5].

i) Direct Numerical Simulation (DNS)

ii) Large Eddy Simulation (LES)

iii) Reynolds-Averaged Navier-Stokes (RANS)

A LES model is chosen since the Re number range to be considered indicates that the flow is fully turbulent. Its adoption responds mainly to the computed large-scales, associated to the coherent structures developed due to the projectile motion. As already stated, the smaller scales are not solved but they are modeled, regarding that its influence over other scales is related to energy transfers [6]. In this work, the CFD is applied to determine the aerodynamic coefficients by using a commercial CFD code called FLUENT which solves the governing equa-

"Corresponding author. 
tions of the flow motion using a technique of finite volume and takes the meshed computational domain from a pre-processor program called ICEM-CFD in which the model of the projectile is drawn and the computational domain is generated and meshed into cells. The implicit segregated structured grid solver was used in these investigations. Second order upwind discritization was used for the flow variables and turbulent viscosity equations, furthermore a comparison with experimental data was performed. The objective of the present study is to provide a methodology for the determination of the aerodynamic coefficients of a projectile of given geometry using the more recent advances in computational fluid dynamic technique. It is convenient to make use of the more powerful packages and the higher computational capacities that are available today.

\section{Computational Fluid Dynamics}

\subsection{Model Geometry}

The full scale $50 \mathrm{~mm}$ projectile with a tapered leading and trailing fin edge and sharp nose tip was modeled in the CFD simulations Figure 1 [7], in order to determine the aerodynamic coefficients. The projectile was 241 $\mathrm{mm}$ long. The nose was tangent ogive. The cylinder body was $111 \mathrm{~mm}$ long. The fins were $6.35 \mathrm{~mm}$ thick with, a $38.1 \mathrm{~mm}$ span, a $76.2 \mathrm{~mm}$ root chord and a $12.7 \mathrm{~mm}$ tip chord. The fin trailing edge was flush with the projectile base.

\subsection{Grid Generation}

The grids for the geometry investigated were obtained from ICEM-CFD software as the numerical grids had been previously constructed each grid was structured and contained mostly hexahedral cells. The projectile contained approximately 1.15 million cells. The computational domain was extended to be 3 times length downstream far from projectile base, 2 times length around the projectile, and $3 / 4$ times length upstream far from projectile nose Figure 2.

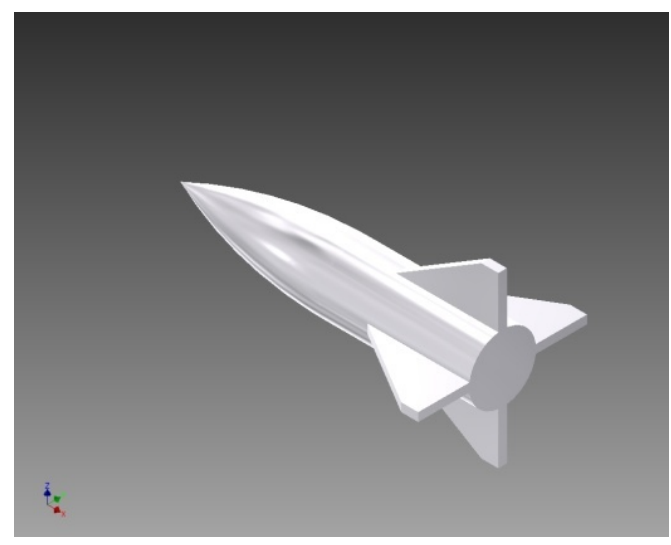

Figure 1. Projectile geometry.
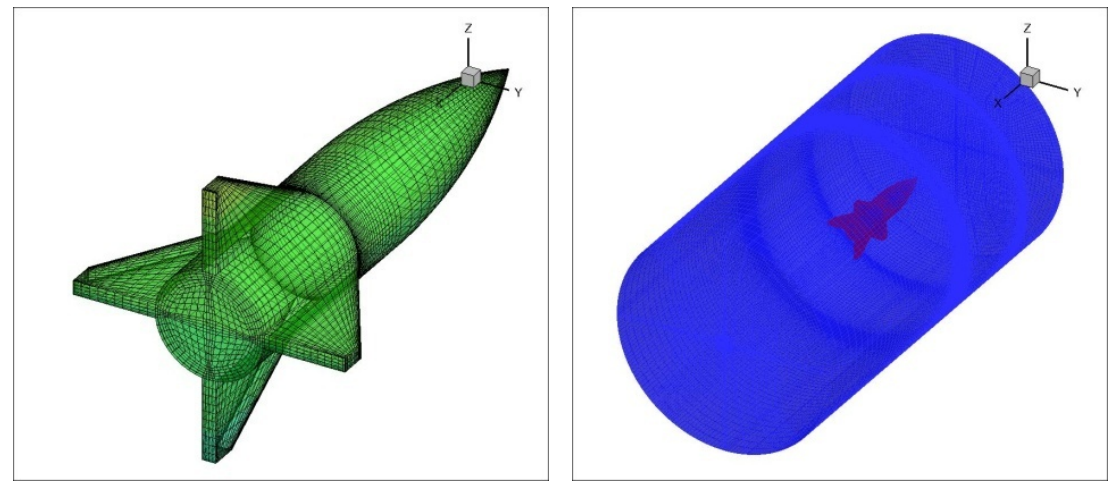

Figure 2. Meshed projectile and computational domain. 


\subsection{Solver}

The CFD is applied to determine the aerodynamic coefficients by using a commercial CFD code called FLUENT which solves the governing equations of the flow motion using a technique of finite volume. The Navier-Stokes equation is numerically solved with a Large Eddy Simulation (LES) method, with the Sub-Grid Scale (SGS) solved by Smagorinsky-Lilly model. In LES techniques, large eddies are resolved directly, while small eddies are modeled. Large Eddy Simulation (LES) thus falls between DNS and RANS in terms of the fraction of the resolved scales. Resolving only the large eddies allows one to use much coarser mesh and larger times-step sizes in LES than in DNS. However, LES still requires substantially finer meshes than those typically used for RANS calculations [8]. In addition, LES has to be run for a sufficiently long flow-time to obtain stable statistics of the flow being modeled; therefore, high-performance computing (e.g., parallel computing) is a necessity for LES as in the present work. The momentum balance equations are solved with an "effective" kinematic viscosity $v_{e}=v+v_{t}$, which is the sum of the molecular part calculated as $v=\mu / \rho$, plus a "turbulent" one $v_{t}$ [9]. The SGS stresses are approximated by second order Roe scheme.

\subsection{Boundary Conditions}

The far field boundary (including the inflow and outflow boundaries) is set to pressure far field density-based inflow/outflow. This boundary condition is a characteristic type that allows the solver to determine the conditions at the far field boundary (inflow, supersonic outflow) and either implicitly sets the boundary condition to free stream conditions (inflow, supersonic outflow). Free stream pressure and temperature are set to 1 atm and $300 \mathrm{~K}$, respectively. Density is then calculated from the perfect gas assumption. Velocity remained fixed at Mach number 1.5. Angle of attack is taken as $0^{\circ}$ and $10^{\circ}$. For the projectile body and fins, when present, the boundary condition is set to be a no slip, adiabatic wall.

\section{Results and Discussion}

Results for case of free stream Mach number $M=1.5$ and angle of attack $\alpha=0^{\circ}, 10^{\circ}$ will be analyzed in order to understand the three-dimensional unsteady compressible flow around a body tail projectile.

\subsection{Drag and Lift Convergence History}

Figure 3 and Figure 4 show the history of the drag and lift coefficient respectively for the two cases $\left(\alpha=0^{\circ}\right.$ and $\alpha=10^{\circ}$ ) with the flow time. For $\alpha=0^{\circ}$ drag coefficient had a constant value after about $0.10 \mathrm{~ms}$ while the lift coefficient had a constant value after about $0.15 \mathrm{~ms}$, furthermore the final total drag coefficient obtained is about 0.38 while the final lift coefficient obtained is about 0.38 . For $\alpha=10^{\circ} \mathrm{drag}$ coefficient had a constant value after about $0.30 \mathrm{~ms}$ while the lift coefficient had a constant value after about $0.31 \mathrm{~ms}$, furthermore the final total drag coefficient obtained is about 0.61 while the final lift coefficient obtained is about 0.88 . As the angle of attack increases, the drag and lift coefficients increase.

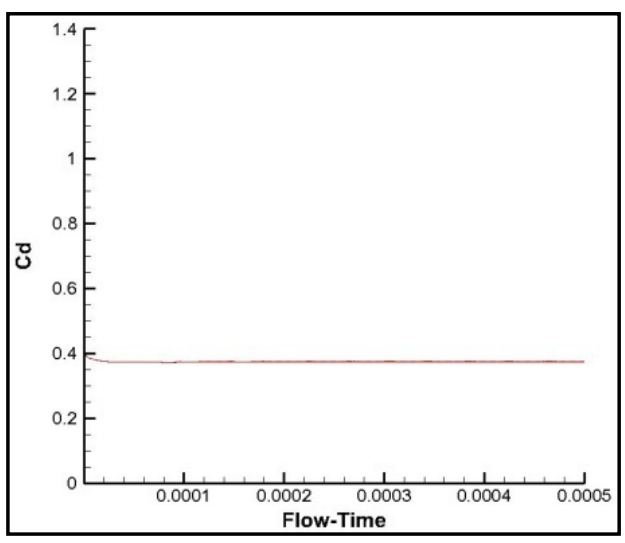

(a)

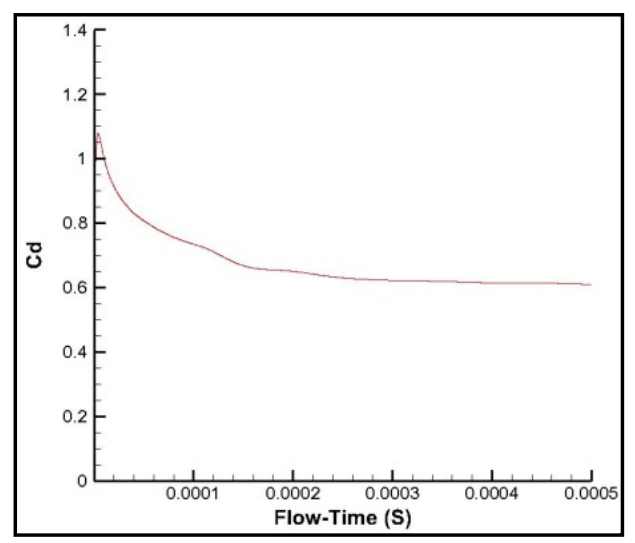

(b)

Figure 3. Drag coefficient convergence history for $M=1.5$. (a) $\alpha=0^{\circ}$; (b) $\alpha=10^{\circ}$. 


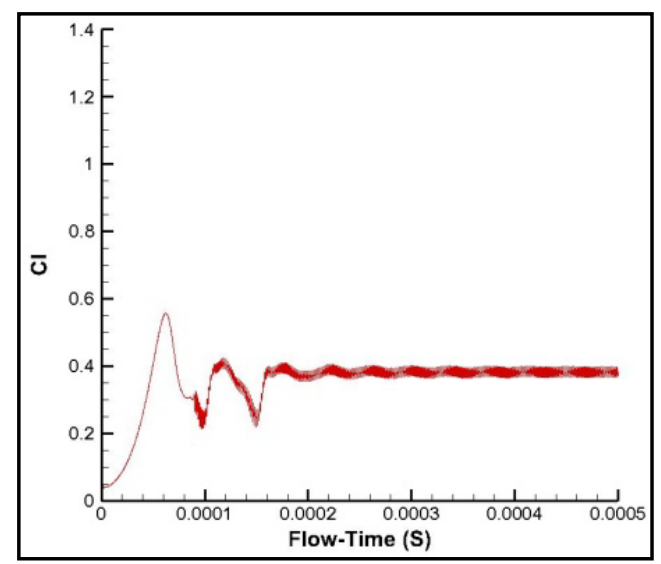

(a)

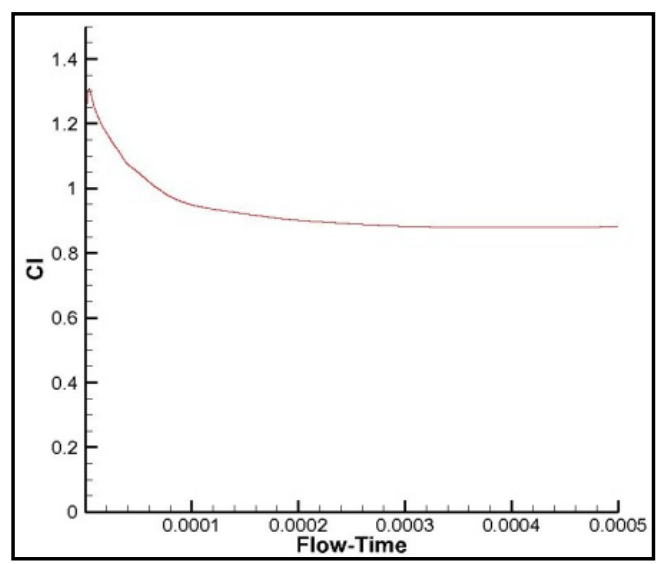

(b)

Figure 4. Lift coefficient convergence history for $\mathrm{M}=1.5$. (a) $\alpha=0^{\circ}$; (b) $\alpha=10^{\circ}$.

\subsection{Contours of Static Pressure Distribution}

From Figure 5 it can be noticed that there is no more symmetry distribution of static pressure contours between upper and lower half of the symmetry plane for $\alpha=10^{\circ}$. The region in which the value of static pressure is lower than the free stream pressure has a large area at the upper half of the symmetry plane. rather than for $\alpha=0^{\circ}$ the symmetry distribution of static pressure contours between upper and lower half of the symmetry plane is cleared.

\subsection{Contours of Mach Number and Velocity Vector Distribution}

Also, from Figure 6 and Figure 7 the Mach number distribution was not symmetric between upper and lower half of the symmetry plane, the boundary layer build up in the upper half is greater than the lower half that is because of the presence of the angle of attack. Figure 8 shows the Mach number distribution behind the base of the body. The wake region, in which the Mach number is minimum value, is clear from the figure. Figure 9 shows the velocity vector distribution behind the base of the body, in which the vortices are clearly large for $\alpha=$ $10^{\circ}$.

\subsection{Comparison between CFD Simulation and Experimental Drag Coefficient}

In Exterior Ballistics, the specialty related to projectile flight studies, the drag coefficient curves take a fundamental rule for different applications, such as the generation of firing tables. These curves can be obtained also from tests inside wind tunnels or theoretical calculus. In the first case, the interferences between the projectile or its model and the tunnel walls affect the precision of results; in the second, the hypothesis adopted could move the results away from the real behavior. Because of the limitations of the methods mentioned before, a different way for the making up of these curves is the identification of the aerodynamic properties of an object from flight test over a real specimen. Figure 10 shows the Comparison between drag coefficient curves obtained by CFD simulations for $0^{\circ}$ angle of attack and Mach number of 1.5, 2, and 3 with the drag curves obtained from [10], (Axial Force Coefficient vs. Mach number, the baseline data). From Figure 10, the drag coefficient decreased linearly with Mach number and shows a good agreement with the baseline tested data.

\section{Conclusion}

3-D unsteady flow CFD simulation around a body tail projectile model were performed at a supersonic Mach number of 1.5 and angles of attack of $0^{\circ}$ and $10^{\circ}$ using a commercial CFD code called (FLUENT 6.3.26) which solves Navier-Stokes equation with a Large Eddy Simulation (LES) method. Sub-Grid Scale (SGS) solved by Smagorinsky-Lilly model, Roe-FDS scheme and a second order discretization were used in this work. A comparison were performed for $\alpha=0^{\circ}$ and Mach number of 1.5, 2, and 3. These 3-D unsteady CFD investigations have provided fundamental understanding of flow field around a body. 


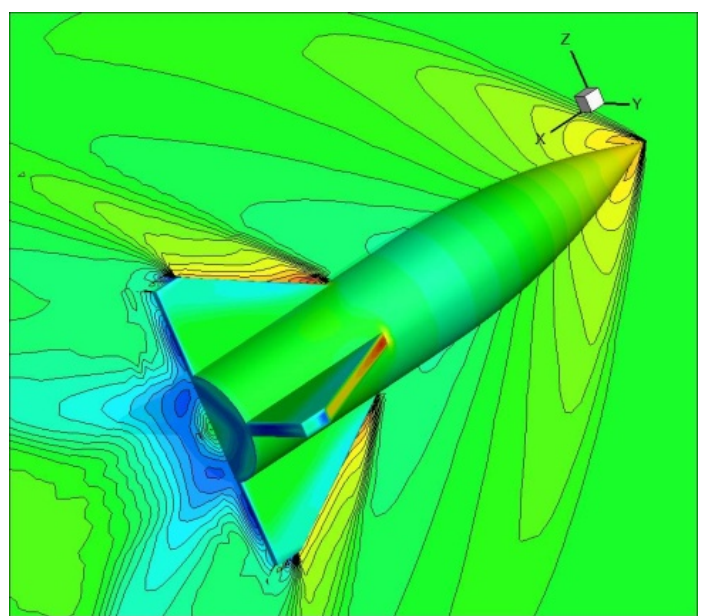

(a)

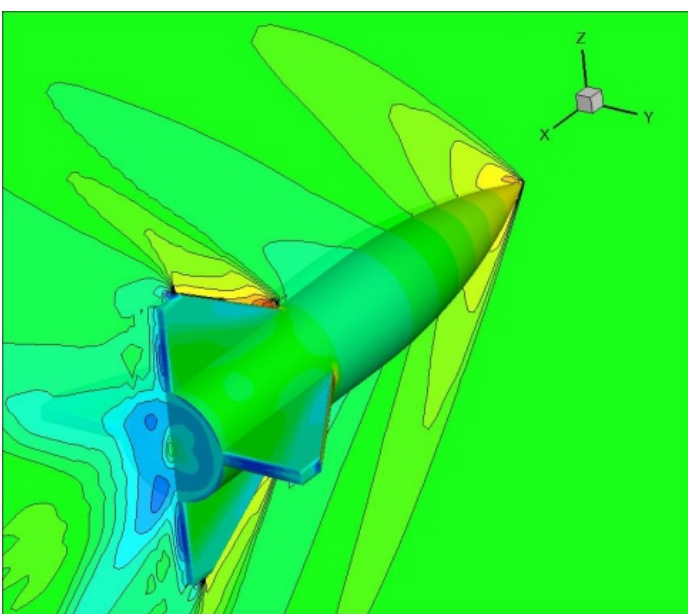

(b)

Figure 5. Contours of static pressure distribution for $\mathrm{M}=1.5\left\{(\mathrm{a}) \alpha=0^{\circ}\right.$ and (b) $\left.\alpha=10^{\circ}\right\}$.

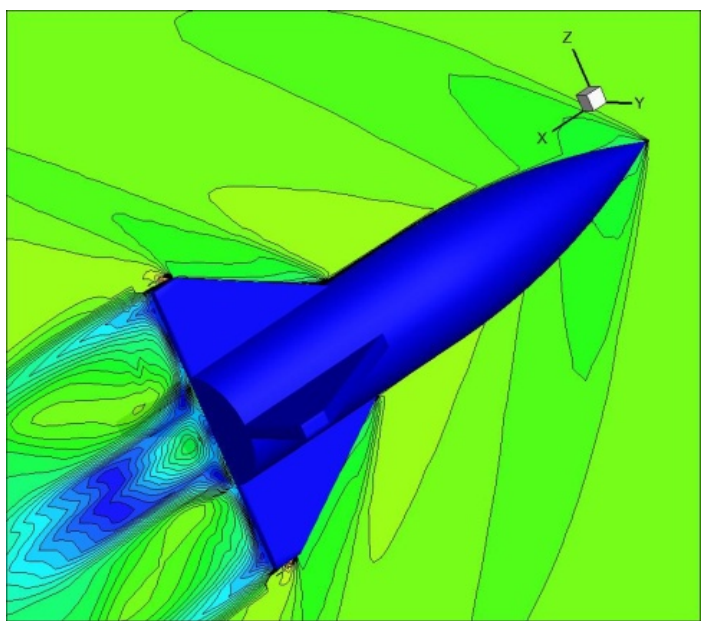

(a)

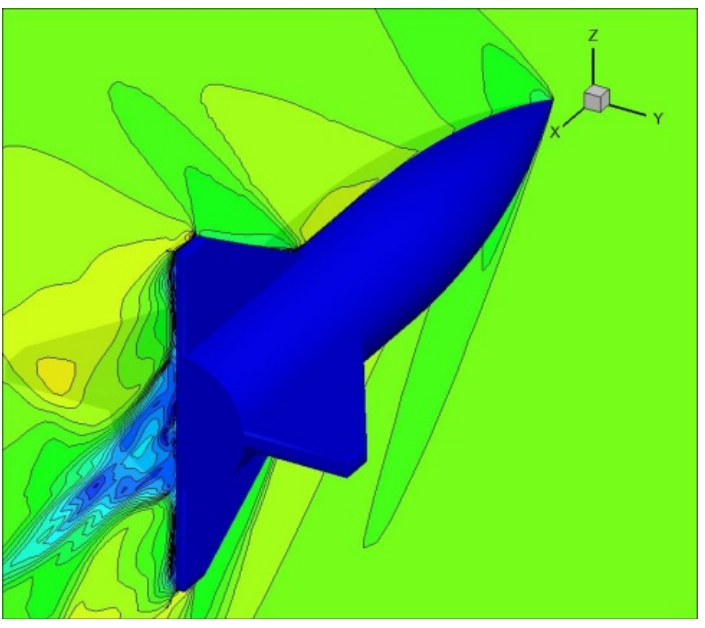

(b)

Figure 6. Mach number distribution for $\mathrm{M}=1.5\left\{(\mathrm{a}) \alpha=0^{\circ}\right.$ and (b) $\left.\alpha=10^{\circ}\right\}$.

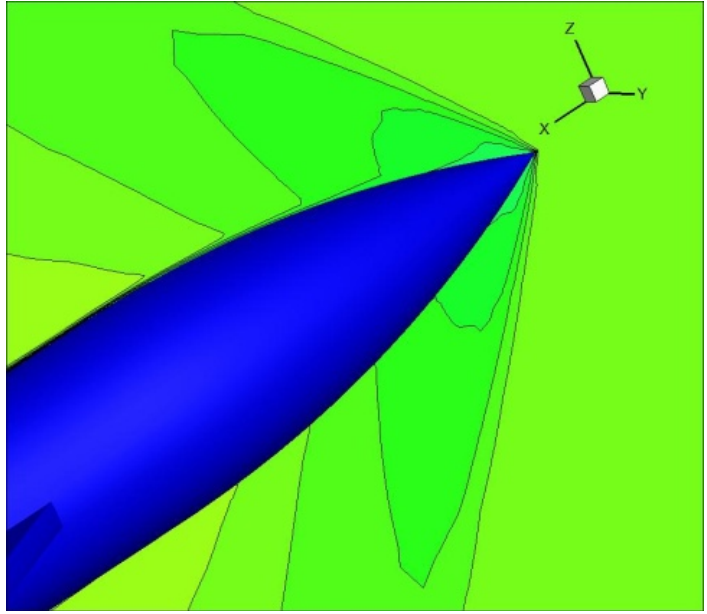

(a)

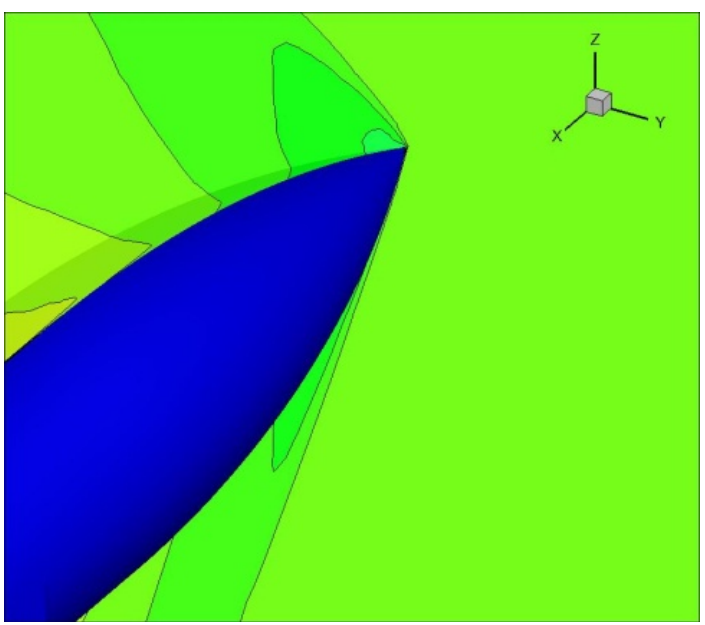

(b)

Figure 7. Mach number distribution nose section for $\mathrm{M}=1.5$ (a) $\alpha=0^{\circ}$ and (b) $\left.\alpha=10^{\circ}\right\}$. 


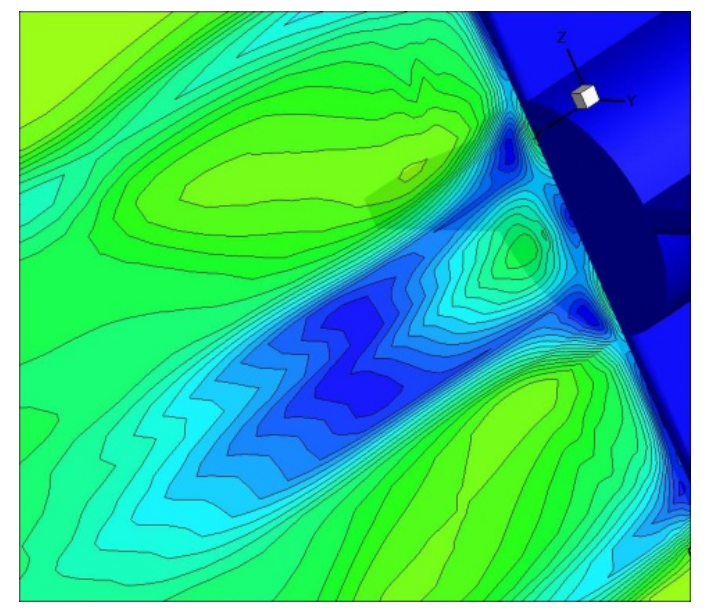

(a)

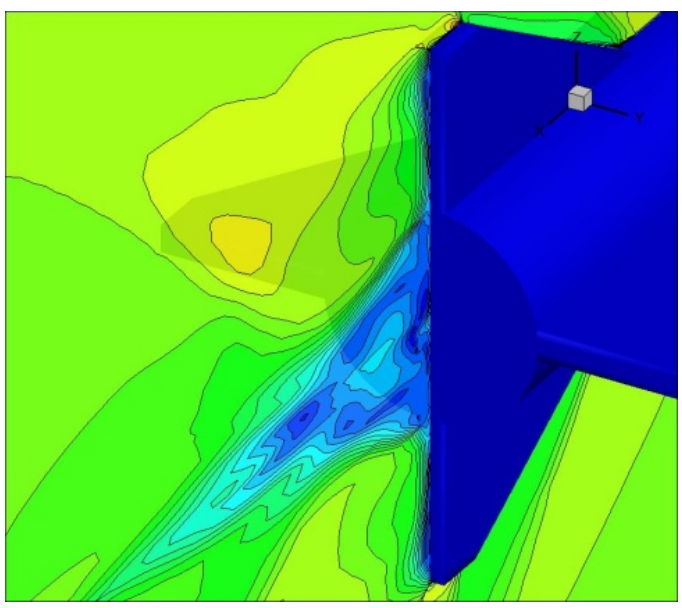

(b)

Figure 8. Mach number distribution base section for $M=1.5\left\{\right.$ (a) $\alpha=0^{\circ}$ and (b) $\left.\alpha=10^{\circ}\right\}$.

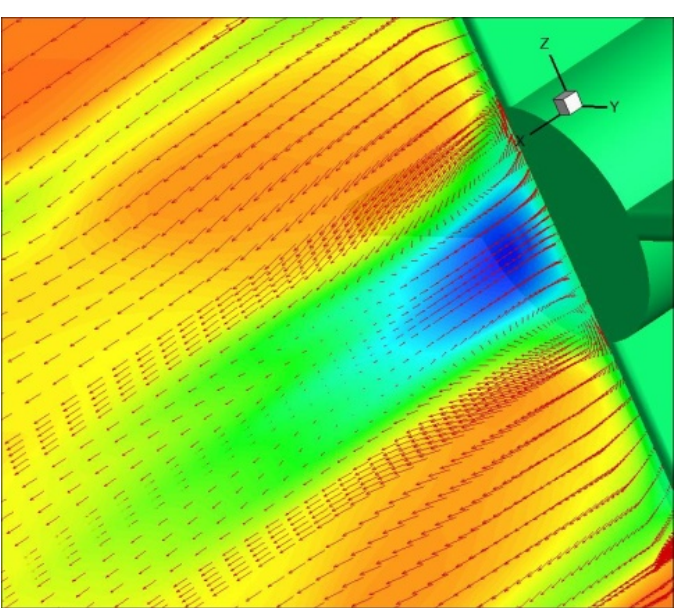

(a)

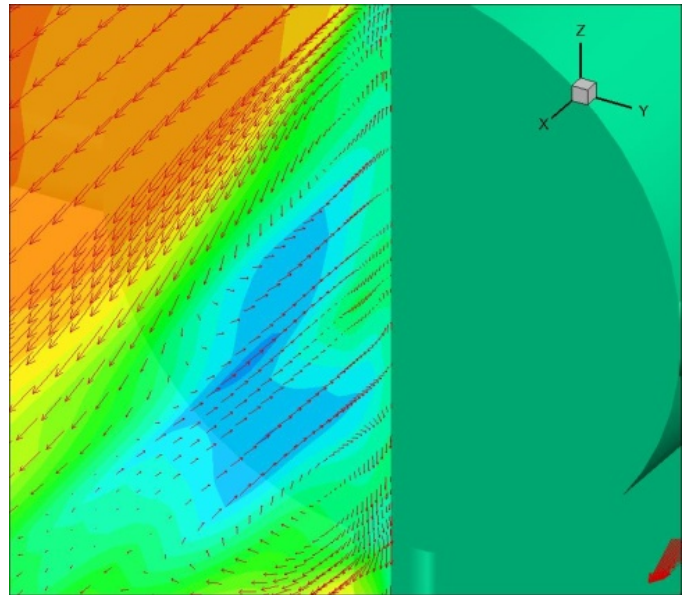

(b)

Figure 9. Velocity distribution base section for $\mathrm{M}=1.5\left\{(\mathrm{a}) \alpha=0^{\circ}\right.$ and (b) $\left.\alpha=10^{\circ}\right\}$.

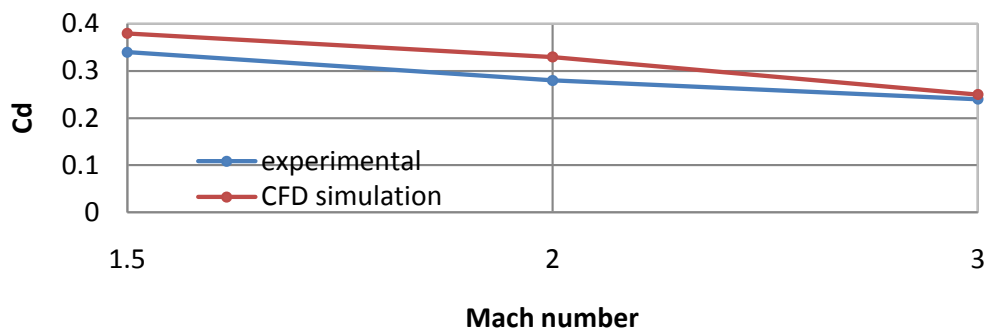

Figure 10. Comparison between CFD simulation and experimental drag coefficient for $\alpha=0^{\circ}[10]$.

\section{Acknowledgements}

This work supported by the Foundation of Key Laboratory of Transient Physics (9140C300205110C30), China.

\section{References}

[1] Sahu, J. (1991) Transonic Navier-Stokes Computations for a Spinning Body of Revolution. Tech. Rep. ARL-TR-3265, US Army Research Laboratory. 
[2] Weinacht, P. (2003) Prediction of Projectile Performance, Stability, and Free-Flight Motion Using Computational Fluid Dynamics. Tech. Rep. ARL-TR-3015, Army Research Laboratory.

[3] Townsend, A.A. (1980) The Structure of Turbulent Shear Flow. 2nd Edition, Cambridge Univ. Press.

[4] Rodi, W. (2006) DNS and LES for Some Engineering Flows. Fluid Dynamics Research, 38, 145-173. http://dx.doi.org/10.1016/j.fluiddyn.2004.11.003

[5] Sagaut, P. (2001) Large Eddy Simulation for Incompressible Flows, an Introduction. Springer, Berlin.

[6] Calo, V. (2005) Residual-Based Multiscale Turbulence Modeling: Finite Volume Simulations of Bypass Transition. Ph.D. Thesis, Stanford University.

[7] Massey, K.C., McMichael, J., Warnock, T. and Hay, F. (2005) Mechanical Actuators for Guidance of a Supersonic Projectile. 23rd Applied Aerodynamics Conference, AIAA 2005-4970.

[8] Chapman, D.R. (1979) Computational Aerodynamics Development and Outlook. AIAA Journal, 17, 1293-1313. http://dx.doi.org/10.2514/3.61311

[9] Smagorinsky, J. (1963) General Circulation Experiments with the Primitive Equations: I. the Basic Equations. Monthly Weather Review, 91, 99-164. http://dx.doi.org/10.1175/1520-0493(1963)091<0099:GCEWTP>2.3.CO;2

[10] Silton, S.I. (2004) Mechanical Actuators for Guidance of a Supersonic Projectile. 22nd Applied Aerodynamics Conference and Exhibit, AIAA 2004-5195. 